\title{
Optimising the oil phases of aluminium hydrogel-stabilised emulsions for stable, safe and efficient vaccine adjuvant
}

\author{
Lili Yuan ${ }^{1}$, Xiao-Dong Gao $(\bowtie)^{1}$, Yufei Xia $(\bowtie)^{2,3,4}$ \\ 1 Key Laboratory of Carbohydrate Chemistry and Biotechnology, Ministry of Education, School of Biotechnology, Jiangnan University, \\ Wuxi 214122, China \\ 2 State Key Laboratory of Biochemical Engineering, Institute of Process Engineering, Chinese Academy of Sciences, Beijing 100190, China \\ 3 University of Chinese Academy of Sciences, Beijing 100049, China \\ 4 Innovation Academy for Green Manufacture, Chinese Academy of Sciences, Beijing 100190, China
}

(C) Higher Education Press 2022

\begin{abstract}
To increase antibody secretion and dose sparing, squalene-in-water aluminium hydrogel (alum)stabilised emulsions (ASEs) have been developed, which offer increased surface areas and cellular interactions for higher antigen loading and enhanced immune responses. Nevertheless, the squalene (oil) in previous attempts suffered from limited oxidation resistance, thus, safety and stability were compromised. From a clinical translational perspective, it is imperative to screen the optimal oils for enhanced emulsion adjuvants. Here, because of the varying oleic to linoleic acid ratio, soybean oil, peanut oil, and olive oil were utilised as oil phases in the preparation of aluminium hydrogel-stabilised squalene-in-water emulsions, which were then screened for their stability and immunogenicity. Additionally, the underlying mechanisms of oil phases and emulsion stability were unravelled, which showed that a higher oleic to linoleic acid ratio increased anti-oxidative capabilities but reduced the long-term storage stability owing to the relatively low zeta potential of the prepared droplets. As a result, compared with squalene-in-water ASEs, soybean-in-water ASEs exhibited comparable immune responses and enhanced stability. By optimising the oil phase of the emulsion adjuvants, this work may offer an alternative strategy for safe, stable, and effective emulsion adjuvants.
\end{abstract}

Keywords pickering emulsion, vaccine adjuvant, alumstabilised emulsion, oleic to linoleic acid ratio, stability

Received July 16, 2021; accepted September 23, 2021

E-mails: xdgao@jiangnan.edu.cn (Gao X), yfxia@ipe.ac.cn (Xiao Y)

\section{Introduction}

Vaccines, such as human papillomavirus vaccine [1], hepatitis B vaccine [2], and polio vaccines [3], have been consistently demonstrated to prevent a wide range of infectious diseases and make an indelible contribution to human health. In addition to live attenuated or inactivated vaccines, there is a tendency to extract only the antigenic components of the pathogens, such as purified proteins, polysaccharides, synthetic peptides, and nucleic acids [4-7]. With simpler structures and higher safety profiles, subunit vaccines were preferred for a quick response to the prevailing pandemic but were at the expense of immunogenicity, as pathogenic structures or components had been removed [8]. Therefore, safe, and effective adjuvants have become increasingly important to stimulate the immune response and reduce the vaccine dosage required for widespread vaccination.

As the approved adjuvant, squalene-in-water emulsions, such as MF59 for influenza vaccines [9], and Adjuvant System 03 for the highly pathogenic Asian avian influenza A virus vaccines [10], have been found to increase antibody secretions for enhanced immune protection. The emulsion droplets activate the immunocytes at the injection sites, which then release antigen-presenting cells (APCs) attracting chemokines and cytokines, completing the immune-amplification loop responsible for enhanced antigen uptake and immune activation [11]. Typically, surfactants are used as stabilisers to reduce the interfacial tension between water and squalene phases [12]. In contrast, surfactant-stabilised interphases form hydration layers that prevent the droplets from adhering to the cell membranes or interacting with antigens. As a result, surfactant-stabilised emulsions are rarely internalised by APCs, making it difficult to manipulate antigen delivery to activate cellular immunity. 
In this study, we developed a particle-stabilised emulsion (Pickering emulsion) to replace surfactants with poly lactic-co-glycolic acid [13], aluminium hydrogel (alum) [14], or albumins [15]. In comparison with conventional emulsions, particle-stabilised interphases provide greater surface areas and intercellular gaps that enhance antigen loading and cellular interactions. Additionally, Pickering emulsion droplets exhibit superior mechanosensing capability and stability [16]. These advantages facilitate threedimensional contact between APCs, as well as the stimulation of humoral and cellular adaptive immunity. However, previous attempts have concentrated on squalene. Even with antioxidants such as tocopherol, it has been shown that squalene is easily oxidised in the main oil phase [17]. Therefore, a series of aldehydes and ketones were generated, which may cause adverse side effects (e.g., local swelling or inflammation) [18] and accelerate the oxidation process, impeding the immunogenicity and safety profile of the emulsion adjuvants. Furthermore, the inter-particle gaps of Pickering emulsions exposed the oil core to the external environment more than the surfactantcovered emulsions, increasing the contact area with the external environment and accelerating the oxidative process. Hence, optimal oils with high stability are required for enhanced immune efficiency, safety, and clinical translation of emulsion adjuvants.

To this end, we optimised the oil phase of the squalenein-water aluminium hydrogel (alum)-stabilised emulsions (ASEs). As a mixture of fatty acids, the approved oils have distinct compositions, such as saturated fatty acids, oleic acid, linoleic acid, linolenic acid, etc. [19-21]. Among them, the oleic to linoleic acid $(\mathrm{O} / \mathrm{L})$ ratio may have major implications on the antioxidative ability, which, in turn, would affect the stability and immunogenicity of the prepared emulsion adjuvants. In this study, clinically approved oils with varying $\mathrm{O} / \mathrm{L}$ ratios were used to prepare a series of ASEs, including soybean oil $(\mathrm{O} / \mathrm{L}$ ratio $=1: 2.75)$ [22], peanut oil $(\mathrm{O} / \mathrm{L}$ ratio $=1.3: 1)[23]$, and olive oil $(\mathrm{O} / \mathrm{L}$ ratio $=4: 1)[24]($ Table 1$)$. The stability of the emulsions was assessed by measuring their size, charge, and degree of oil oxidation. The effectiveness of the antigen adsorption and reservoir effect of the antigen, as well as the antibody, memory $\mathrm{T}$ cell, and cytokine profiles, were also evaluated. By comparing the stability and immunogenicity of the prepared ASEs, this study elucidated the underlying relationship between the oil phase $\mathrm{O} / \mathrm{L}$ ratio and emulsion stability, shedding light on the optimal oil phase for stable, safe, and effective emulsion adjuvants.

Table 1 Selected oils for ASEs optimisations

\begin{tabular}{lccccc}
\hline Oil type & $\begin{array}{c}\text { Saturated } \\
\text { fatty acid/\% }\end{array}$ & $\begin{array}{c}\text { Oleic } \\
\text { acid/\% }\end{array}$ & $\begin{array}{c}\text { Linoleic } \\
\text { acid/\% }\end{array}$ & $\begin{array}{c}\text { O/L } \\
\text { ratio }\end{array}$ & Ref. \\
\hline Soybean oil & 17.0 & 20.0 & 55.0 & $1: 2.75$ & {$[4]$} \\
Peanut oil & 20.7 & 44.2 & 35.1 & $1.3: 1$ & {$[5]$} \\
Olive oil & 8.9 & 72.5 & 18.0 & $4: 1$ & {$[19]$} \\
\hline
\end{tabular}

\section{Experimental}

\subsection{Chemicals and reagents}

Aluminium hydroxide adjuvant and ELISA kits for cytokine profiles were purchased from the InvivoGen Company. Soybean oil, olive oil, peanut oil, squalene, and ovalbumin (OVA, $45 \mathrm{kDa}$ ) were purchased from the American Sigma Company. Phosphate-buffered saline (PBS, $0.01 \mathrm{~mol} \cdot \mathrm{L}^{-1}, \mathrm{pH}=7.2-7.4$ ), paraformaldehyde, and red blood cell lysate were purchased from Beijing Solarbio Company. Hydrochloric acid (relative molecular weight 36.46 , purity $36.0 \%-38.0 \%$ ), sodium hydroxide, and sodium chloride were purchased from Beijing Chemical Reagent Company. Sodium citrate (relative molecular weight 294.10 , purity $\geqslant 99.0 \%$ ), disodium hydrogen phosphate $\left(\mathrm{Na}_{2} \mathrm{HPO}_{4} \cdot 12 \mathrm{H}_{2} \mathrm{O}\right.$, relative molecular mass 358.14 , purity $\geqslant 99 \%$ ), and sodium dihydrogen phosphate $\left(\mathrm{NaH}_{2} \mathrm{PO}_{4} \cdot 2 \mathrm{H}_{2} \mathrm{O}\right.$, relative molecular mass 156.01 , purity $\geqslant 99 \%$ ) were purchased from Sinopharm Chemical Reagent. Fluorescent gallium was prepared by the American Thermo Scientific Company. The MicroBCA protein quantification kit was purchased from Thermo Fisher Scientific (USA). RPMI medium 1640 and fetal bovine serum were purchased from Gibco Technologies. BALB/c female mice were purchased from Beijing Vital River Animal Laboratories. Aspartate aminotransferase (AST), alanine aminotransferase (ALT), blood urea nitrogen (BUN), lactate dehydrogenase (LDH), and alanine aminotransferase (ALP) were purchased from Toshiba. CD3 monoclonal antibody (145-2C11), PE-Cy7, CD44 monoclonal antibody (IM7), PerCP-Cyanine5.5, CD62L (L-Selectin) monoclonal antibody (MEL-14), and APC were purchased from TONCO Bio.

\subsection{Preparation and optimisations of ASEs}

The ASEs were prepared by single-step sonication (Branson Digital Sonifier, interval time $=4 \mathrm{~s}$ ) of commercial aluminium hydroxide and the oils. Subsequently, the emulsions were optimised for the smallest size distribution according to the particle concentration and oil phase volume. Meanwhile, a similar size distribution was optimised to the buffer type, buffer $\mathrm{pH}$, ultrasonic power, ultrasonic time, and ultrasonic temperature, respectively. Afterwards, a dynamic light scattering (DLS) analyser (Malvern) was employed to measure the zeta potential and particle size at $25^{\circ} \mathrm{C}$. The experiments were performed in triplicate and obtained similar results.

The content of the ASE endotoxin was determined using the limulus reagent test method. The assessment was conducted according to the manufacturer's protocol [25]. We prepared the ASEs standard reagents and other necessary solutions; further, $100 \mu \mathrm{L}$ of the standard and sample were added to the endotoxin-free vials, then $100 \mu \mathrm{L}$ of limulus amebocyte lysate was added to the upper tubes 
and incubated at $37^{\circ} \mathrm{C}$ for $30 \mathrm{~min}$. In each tube, $100 \mu \mathrm{L}$ of chromogenic substrate was added and incubated at $37^{\circ} \mathrm{C}$ for $6 \mathrm{~min}$. Subsequently, $500 \mu \mathrm{L}$ color-stabilizer \#1, $500 \mu \mathrm{L}$ color-stabilizer \#2, and $500 \mu \mathrm{L}$ color-stabilizer \#3 were added gradually. Subsequently, $100 \mu \mathrm{L}$ was pipetted into a 96-well plate, the absorbance was measured at 545 $\mathrm{nm}$, and the endotoxin content in the sample was calculated according to the standard curve.

Further, $100 \mu \mathrm{L}$ ASEs were mixed with OVA antigen at room temperature for $30 \mathrm{~min}$. The fluidic OVAs were then collected from the subnatants of OVA/ASEs after centrifugation at $4000 \times g$ for $5 \mathrm{~min}$. The antigen concentrations were determined using a Micro BCA protein assay kit (Thermo-Fisher). The antigen-loading efficiency was calculated using the following equation:

ASE-loading antigen efficiency $=($ total OVA - fluidic OVA) $/$ total OVA $\times 100 \%$

The co-localisation of OVA with the droplets was observed using a laser scanning confocal microscope (SP8 STED 3X, Leica), where alum (green) and OVA (red) were labelled by Lumogallion (Sigma) and Cy5 (Thermo Fisher), respectively.

\subsection{Immunogenicity of the prepared ASEs with varied $\mathrm{O} / \mathrm{L}$ ratio}

To estimate the fluorescence intensity of the antigen at the injection site, the OVA antigens were labelled with Cy5 (Thermo Fisher) and loaded with the prepared ASEs. Afterwards, BALB/c mice (6-8 weeks old, female, Beijing Vital River Animal Laboratories) were intramuscularly administrated with $100 \mu \mathrm{L}$ ASEs/OVA at the thigh. The antigen reservoir effect was evaluated by the fluorescence decay at the injection sites, which were traced through the in vivo imaging system FX Pro (Kodak). The fluorescence imaging settings were as follows: excitation wavelength, $650 \mathrm{~nm}$; emission wavelength, $700 \mathrm{~nm}$.

On Day 0, the BALB/c mice (6-8 weeks old, female, Beijing Vital River Animal Laboratories) were intramuscularly administrated with $100 \mu \mathrm{L}$ ASEs/OVA at the thigh. For humoral responses (serum antibody titer), serum samples were collected 28 days after the first administration. In the ELISA analysis of immunoglobulin G (IgG), 96-well plates were coated at $4{ }^{\circ} \mathrm{C}$ overnight with a coating buffer $\left(50 \mathrm{mmol} \cdot \mathrm{L}^{-1} \mathrm{Na}_{2} \mathrm{CO}_{3}-\mathrm{NaHCO}_{3} \mathrm{pH} 9.6\right.$, OVA $2 \mu \mathrm{g}$ per well). The next day, the plates were washed three times with phosphate buffered saline containing $0.05 \%(\mathrm{w} / \mathrm{v})$ Tween 20 (PBST), and then blocked with blocking buffer $\left(0.5 \%(\mathrm{w} / \mathrm{v}) \mathrm{BSA}\right.$ in PBST) for $1 \mathrm{~h}$ at $37^{\circ} \mathrm{C}$. Subsequently, a two-fold dilution series, beginning at an initial 100-fold dilution, was used to determine the concentration of OVAspecific IgG. The samples were incubated at $37{ }^{\circ} \mathrm{C}$ for $1 \mathrm{~h}$. Afterwards, the plates were washed six times with PBST. The secondary antibody (anti-mouse IgG-horseradish peroxidase, 1:50000 dilution, Abcam) was then added and incubated at $37{ }^{\circ} \mathrm{C}$ for $30 \mathrm{~min}$. Afterwards, the substrate tetramethylbenzidine (Abcam) was added to trigger the chromogenic reactions with the horseradish peroxides under dark light, which was subsequently stopped 15 min later. To collect the data, an Infinite 200 PRO (TECAN) was employed to obtain the $\mathrm{OD}_{450}$ values. The titers were determined as the $\mathrm{OD}_{450}$ of the dilutions that were twice the mean of the background (negative serum).

On Day 28, the splenocytes were isolated by mincing the spleen through 75-mesh cell strainers. Additionally, lysis buffer ( $0.9 \%$ ammonium chloride, eBioscience) was added to the eliminate erythrocyte interference. After separation $(500 \times g, 5 \mathrm{~min})$, a single-cell suspension was obtained. After the single-cell suspension was obtained, the splenocytes $\left(4 \times 10^{6}\right.$ cells $\left.\cdot \mathrm{mL}^{-1}\right)$ were pulsed with the antigens $\left(4 \mu \mathrm{g} \cdot \mathrm{mL}^{-1} \mathrm{OVA}\right)$ and co-incubated at $37^{\circ} \mathrm{C}$ for $48 \mathrm{~h}$. Afterwards, the supernatants of the cells were collected by centrifugation $(500 \times g, 5 \mathrm{~min})$. The cytokine profile was assessed by the concentrations of IFN- $\gamma$ and IL-4 in the collected supernatants, using ELISA kits with an ELISA plate reader (Infinite M200, TECAN). The cells were collected and stained with fluorescent antibodies: 145-2C11, PE-Cy7, IM7, PerCP-Cyanine5.5, MEL-14, and APC (all from TONCO Bio). For immune memory, CD44 ${ }^{\text {high }} \mathrm{CD} 62 \mathrm{~L}^{\text {high }}$ and $\mathrm{CD} 44^{\text {high }} \mathrm{CD} 62 \mathrm{~L}^{\text {low }}$ were used to identify the central and effector memory $\mathrm{T}$ cells, respectively. The antibody dilution for the flow cytometry staining was performed according to the manufacturer's instructions. Normally, 1:100 antibody dilution was employed for 106 cells. Each experiment was repeated three times and similar results were obtained.

\subsection{Biosafety profile}

On Day 0 and Day 14, the BALB/c mice were intramuscularly injected with ASEs and alum. For local reactions, the quadriceps $(n=6)$ were collected after 14 days of injection. Isolated muscle tissue was fixed overnight in a pre-cooled $4 \%$ paraformaldehyde solution. In addition to embedding in paraffin, tissue sections and hematoxylin-eosin (H\&E) staining were performed for microscopic examination. For systemic toxicities, serum ( $n$ $=6$ ) was collected 28 days after the first vaccination. The toxicity effect was evaluated according to the serum biochemical parameters, including AST, ALT, BUN, LDH, and ALP. Further, the hearts, spleens, livers, lungs, and kidneys were isolated and stained with H\&E. Additionally, we tested the alum concentration in the homogenate of the major organs. Additionally, the cytokine storm was evaluated based on the concentrations of IL-10, IL-8, and IL-6 in the supernatants of the harvested splenocytes.

\subsection{Stability evaluations}

Regarding the storage stability, the prepared ASEs were stored at $4{ }^{\circ} \mathrm{C}, 25^{\circ} \mathrm{C}$, and $37^{\circ} \mathrm{C}$. The size, zeta potential, 
and optical images were monitored from Day 0 to Day 30 . The experiments were performed in triplicate and obtained similar results.

For oxidation stability, $2 \mathrm{~mL}$ of the testing oils and the prepared ASEs were placed in an oxidation stability tester (892 Professional Rancimat, Metrohm). The oxidation induction period time was collected under accelerative conditions $\left(120{ }^{\circ} \mathrm{C}, 20.0 \mathrm{~L} \cdot \mathrm{h}^{-1}\right.$ gas flow rate) [26]. The experiments were performed in triplicate and obtained similar results.

A Likert scale was developed to comprehensively compare the stability of the prepared ASEs [27]. The scale scores demonstrated construct validity through association with constructs of interest: aggregation time of micrographs, size change degree (at room temperature), charge change degree (at room temperature), and oxidation induction period. Samples were collected from a representative experiment $(n=3)$ out of three independent experiments. The scoring principles are listed in Table 2.

Table 2 Marking standard of stability evaluation scale

\begin{tabular}{lcccc}
\hline Score & $\begin{array}{c}\text { Aggregation } \\
\text { time of } \\
\text { micrographs/min }\end{array}$ & $\begin{array}{c}\text { Size change } \\
\text { degree/nm }\end{array}$ & $\begin{array}{c}\text { Zeta potential } \\
\text { change } \\
\text { degree/mV }\end{array}$ & $\begin{array}{c}\text { Oxidation } \\
\text { induction } \\
\text { period/h }\end{array}$ \\
\hline 5 & $>4$ & $<200$ & $<1$ & $>1$ \\
4 & $3-4$ & $200-350$ & $1-2$ & $0.8-1$ \\
3 & $2-3$ & $350-500$ & $2-3$ & $0.6-0.8$ \\
2 & $1-2$ & $500-650$ & $3-4$ & $0.4-0.6$ \\
1 & $<1$ & $>650$ & $>4$ & $<0.4$ \\
\hline
\end{tabular}

\subsection{Ethic statement}

All animals were obtained from the Vital River Animal Laboratories. Their cages were sterile, equipped with laminar airflow hoods, and were arranged in groups of five or fewer in a pathogen-free room. To feed the animals, autoclaved food was used with water. A week of acclimation for mice was guaranteed prior to the experiments. All animal protocols were approved by the Committee on the Ethics of Animal Experiments of the Institute of Process Engineering at the Chinese Academy of Sciences (approval ID: IPEAECA20210402). Additionally, we modified all techniques and procedures to ensure the animals' happiness and minimise their stress. The data are presented as the mean \pm s.e.m. $(n=6)$.

\subsection{Statistical analysis}

All animal studies were performed after randomisation. All values are expressed as mean \pm s.e.m. and the data were analysed by one-way or $t$-test analysis of variance (ANOVA) for comparison of multiple groups using GraphPad Prism 8 software. Statistical significance was set at $p<0.05$.

\section{Results and discussion}

\subsection{Optimisations and characterisations of ASEs}

Here, we prepared a series of ASEs optimisations, including alum concentration, oil phase volume, buffer type, and solution pH (Fig. S1, cf. Electronic Supplementary Material, ESM). Initially, the particle concentration plays a major role in the particle arrangement at the interface between oil and water and the size of the emulsion [28]. We primarily displayed different alum concentrations and detected their sizes by DLS, as shown in Fig. S1(a). As the amount of alum increased, the size of the ASEs first increased and then decreased. To obtain the smallest ASEs, the alum concentration was chosen (2 $\mathrm{mg} \cdot \mathrm{mL}^{-1}$ ). Furthermore, at the same alum concentration, Fig. S1(b) showed that higher oil volumes lead to larger droplet sizes and reduced stability, which is in agreement with previous findings [29].

To optimise the hydration layer of the emulsion droplets, the continuous phase was optimised in terms of the buffer type and $\mathrm{pH}$. Compared with PBS and saline-buffered droplets, the ASEs were smaller in the deionised water and citrate buffer (the fluidic mixture of $100 \mathrm{mmol} \cdot \mathrm{L}^{-1}$ citric acid and sodium citrate, respectively) (Fig. S1(c), Fig. S2, cf. ESM), indicating that the presence of phosphate anions may disrupt the hydration layer by the coordinate effect with alum [30]. The citrate buffer was selected with the narrowest size distribution. In the case of $\mathrm{pH}$, aluminium hydroxide ionisation resulted in low affinity for oil phases at lower $\mathrm{pH}$ values, which made the emulsion difficult to stabilise. Alternatively, alkaline buffers tend to cause an increased agglomeration of droplets. To improve the uniformity, a $\mathrm{pH}$ of 7.4 was selected for the preparation of ASEs (Fig. S1(d)). Furthermore, the different $\mathrm{O} / \mathrm{L}$ ratios of the oil samples resulted in different spreading rates, thereby affecting the energy input necessary to generate oil droplets. The optimal ultrasonic conditions were also evaluated according to the ultrasonic power, ultrasonic temperature, and ultrasonic time (Fig. S1(e-g)).

Subsequently, the efficiency of the ASE-loading antigen on the emulsion surface was evaluated. According to the confocal images, Cy5 labelled antigen (red) and fluorescent gallium-labelled alum (green) co-localised on the emulsion surface, demonstrating the high antigen adsorption of the prepared ASEs (Fig. 1(a)). From a quantitative perspective, the loading efficiency was determined based on the protein concentration in the supernatant. A high percentage of antigen was adsorbed on all ASEs, as shown in Fig. 1(b); only $3 \%$ of the fluidic alum/antigen complex was detected (Fig. S3(b), cf. ESM). Thus, more antigens were adsorbed on the ASEs, and the different oil phases did not affect the adsorption of antigens despite their differing zeta potentials. Collectively, the ASEs were prepared with similar sizes (approximately $1600.00 \pm 71.75 \mathrm{~nm}$ ), PDIs (approximately 
$0.06 \pm 0.04$ ), and antigen adsorption efficiencies (approximately $100 \%$ ), as presented in Table 3 .

Moreover, all formulations tested were within a relatively low endotoxin level range (Fig. S4, cf. ESM). Additionally, no significant differences were observed between the groups. Thus, endotoxin contamination had little impact on the immunogenicity of ASEs with different oil phases.

3.2 Immunogenicity of the prepared ASEs with varied $\mathrm{O} / \mathrm{L}$ ratio

\subsubsection{Comparable antigen depot of the ASEs}

The local reaction at the injection site was first evaluated to determine the immunogenicity of the ASEs. Here, Cy5labelled antigens were adsorbed on the ASEs (Table 3), injected intramuscularly, and traced using an in vivo fluorescence imaging system. Although the oil phases varied, all the ASEs formed strong antigen depots for 5 days, which prolonged the time window for antigen uptake and the activation of recruited immune cells, as shown in Fig. 2. Additionally, there were no significant differences among the ASE groups, indicating that the selected oils had little effect on antigen persistence. The antigen depot effect may be attributed to the dense-packed alum on the $\mathrm{O} / \mathrm{W}$ interphase, conferring a large specific surface area to adsorb large amounts of antigens, which subsequently form an antigen pool to recruit and cultivate the APCs and trigger immune activation [31].

\subsubsection{Humoral immune response}

Humoral responses elicit pathogen-specific immunoglobulins, such as IgM, IgG, and its subtypes, to distinctly recognise antigenic epitopes and neutralise the infections of pathogens systemically [32]. To evaluate the humoral immune responses, the indicated formulations were intramuscularly administered to BALB/c mice on Day 0 and Day 14. Serum was collected 28 days after the first vaccination, and subsequently, the titers of the antigenspecific IgG were determined for the humoral immunity assessment. Comparing the ASE-vaccinated serum with that of alum-vaccinated serum, the IgG titers specific for OVA were 10 times higher $(P=0.00025)$. Furthermore, ASE-soybean and ASE-squalene both induced an increase in IgG titers by 1.14-fold and 1.33-fold, respectively, compared with ASE-peanut $(P=0.97)$ and ASE-olive $(P=$ $0.77)$, indicating stronger humoral immune protection (Fig. 3). (a)
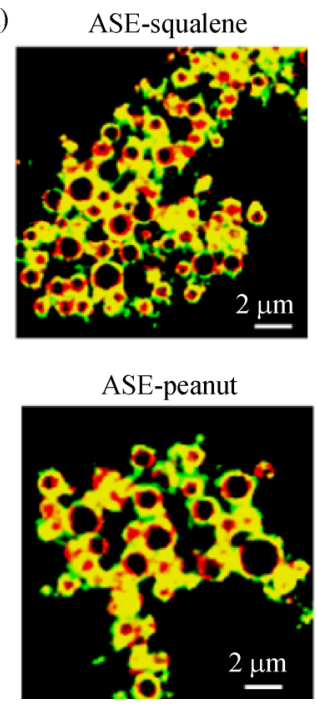

ASE-soybean

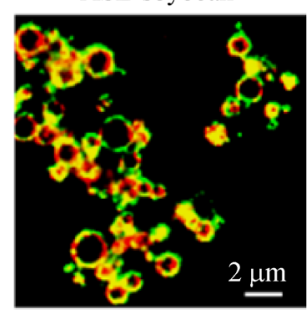

ASE-olive

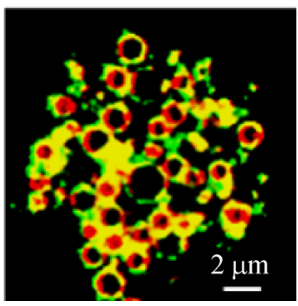

(b)

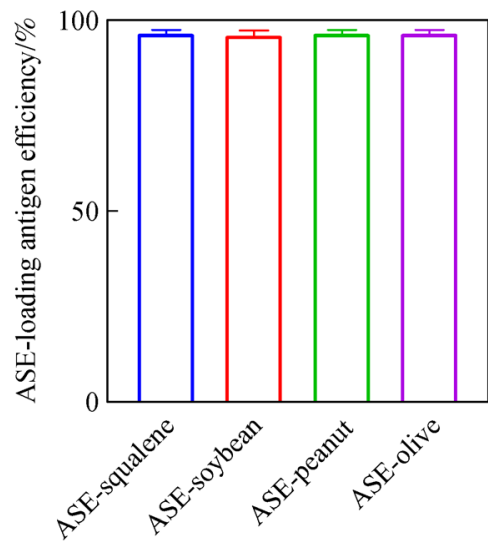

Fig. 1 (a) Confocal images of ASEs and (b) ASE-loading antigen efficiency. OVA and the surface alum were labelled with Cy5 (red) and Lumogallion (green), respectively. Scale bar $=2 \mu \mathrm{m}$. Data were shown as mean \pm s.e.m. $(n=3)$.

Table 3 Optimal formulations and characterisations on the ASEs ${ }^{\text {a) }}$

\begin{tabular}{lcccc}
\hline Item & ASE-squalene & ASE-soybean & ASE-peanut & ASE-olive \\
\hline Ultrasonic power/\% & 50.0 & 50.0 & 50.0 & 20.0 \\
Size/nm & $1682.3 \pm 64.0$ & $1692.3 \pm 102.0$ & $1589.7 \pm 181.3$ & $1497.7 \pm 143.8$ \\
PDI & $0.053 \pm 0.059$ & $0.08 \pm 0.013$ & $0.029 \pm 0.026$ & $0.076 \pm 0.154$ \\
Zeta potential $/ \mathrm{mV}$ & $-15.5 \pm 3.0$ & $-22.3 \pm 2.5$ & $-0.5 \pm 0.2$ & $-8.4 \pm 1.1$ \\
\hline
\end{tabular}

a) Alum: $20 \mathrm{mg} \cdot \mathrm{mL}^{-1}$; oil: $50 \mu \mathrm{L} \cdot \mathrm{mL}^{-1}$; buffer type: citrate, $\mathrm{pH}$ 7.4; ultrasonic temperature: ice-bath; ultrasonic time: $2.0 \mathrm{~min}$. 


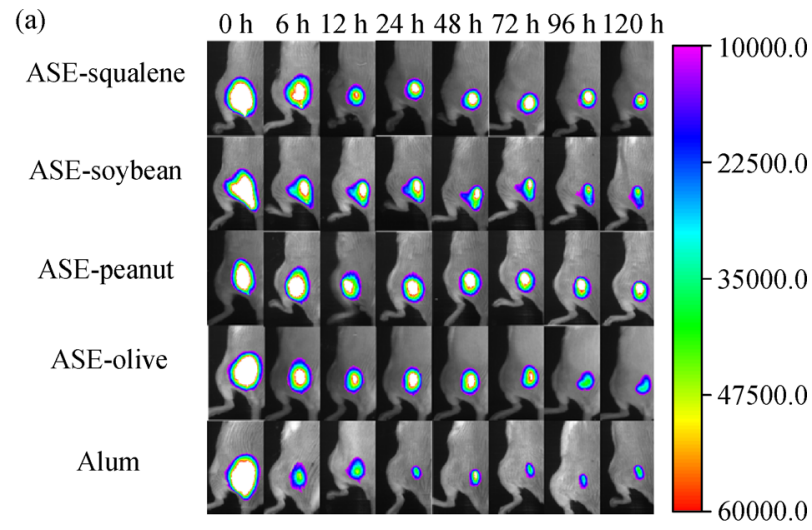

(b)

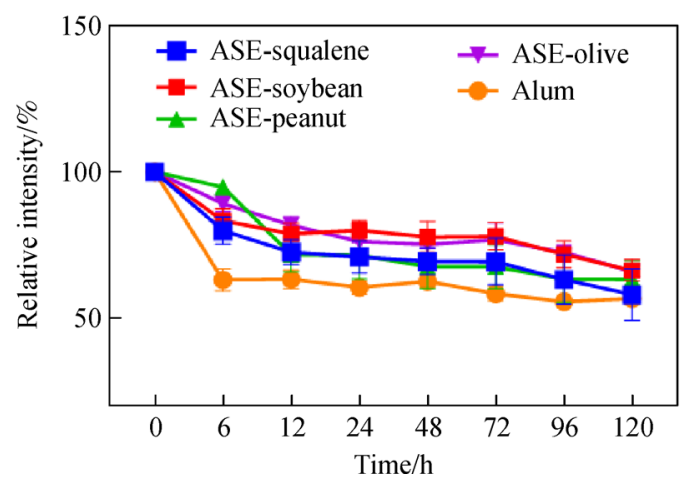

Fig. 2 Antigen reservoir effect: (a) in vivo images and (b) quantitative fluorescent intensity of Cy5-OVA persistence at the injection sites. Data were shown as mean \pm s.e.m. $(n=6)$.

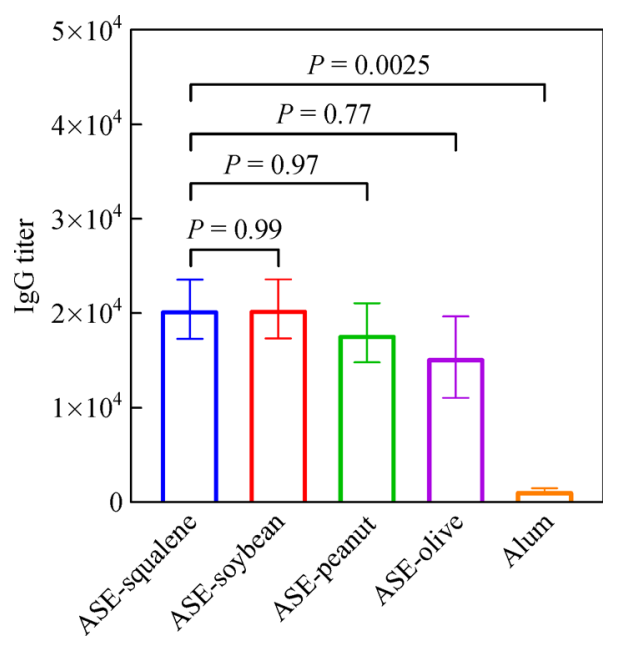

Fig. 3 Serum OVA-specific $\operatorname{IgG}$ titer on Day 28. Data were shown as mean \pm s.e.m. $(n=6)$ and analysed by one-way ANOVA.

\subsubsection{Cellular immune responses}

Cellular immune responses are critical for defence against vital infections, which comprise T-cell responses, such as $\mathrm{CD}^{+}$and $\mathrm{CD} 8^{+} \mathrm{T}$ cells [33]. To verify whether the oil phases had an impact on the cellular immunity, we tested the cytokine profile of ASE/OVA-pulsed splenocytes (Fig. 4). When compared with alum, the ASEs produced $200 \%$ higher IFN- $\gamma$ secretion in the superantigen $(P<0.0001)$, suggesting elevated Th1 (T-Help 1 Cell)mediated immune responses (Fig. 4(a)). Furthermore, ASE-soybean elicited higher IL-4 concentrations than ASE-peanut $(P=0.60)$ (Fig. $4(\mathrm{~b}))$, indicating a potent Th2bias activation (T-Help 2 Cell). Collectively, soybean-oilbased ASEs induced a relatively higher cellular immune response, which may provide comprehensive immunity to pathogen invasion.

\subsubsection{Memory T-cell activations}

Thereafter, the dynamics of the memory $\mathrm{T}$ cells were examined. Among the splenocytes, the effective memory $\mathrm{T}$ cells $\left(\mathrm{CD}^{+} \mathrm{CD} 44^{\text {high }} \mathrm{CD} 62 \mathrm{~L}^{\text {low }}\right)$ potently increased in response to the ASE-formulated vaccinations, suggesting the ability to elicit immediate antiviral responses in pathogen encounters (Fig. 4(c)). Furthermore, ASEsoybean and ASE-squalene induced more memory $\mathrm{T}$ cells than ASE-peanut $(P=0.32)$ and ASE-olive $(P=$ 0.45). Moreover, ASE-soybean elicited higher central memory $\mathrm{T}$ cells among the splenocytes $\left(\mathrm{CD}^{+} \mathrm{CD} 44^{\text {high }}\right.$ CD62L $\left.\mathrm{L}^{\text {high }}\right)$, with $140 \%$ and $120 \%$ increase, in contrast to ASE-olive $(P=0.0004)$ and ASE-squalene $(P=0.056)$, respectively (Fig. 4(d)). Higher proportions indicated a higher immune memory effect to boost the proliferation and differentiation of $\mathrm{T}$ cells against viral infections. In contrast to ASE-peanut and ASE-olive, ASE-soybean induced relatively higher immune activation, which is comparable to ASE-squalene.

\subsection{Biosafety profile}

We then assessed the safety and biocompatibility of the ASEs by assessing the serum biochemicals, as well as histological changes in the vital organs and injection sites. Fourteen days after the first administration, the injection sites showed no apparent side effects, such as inflammation (Fig. 5). Additionally, the tissue distribution analysis of the emulsion droplets indicated that the droplets were hardly distributed in the major organs (Fig. S5, Table S1, cf. ESM) and was retained at the injection sites for cellular infiltration. This indicates the enhanced safety profile of the ASEs [34]. In the case of systemic toxicity, the levels of AST, ALT, BUN, LDH, and ALP on Day 28 showed no significant difference from the untreated group (PBS) and alum group, indicating the limited adverse effects on the circulatory, hepatic, and renal functions (Table 4). Additionally, no evident pathology was found in the vital organs on Day 28, including the heart, liver, spleen, lungs, and kidneys (Fig. 5). Regarding the immunotoxicity (Fig. 6), there were no deviations in the IL-6 and IL-8 secretions observed among the ASE-primed groups, 

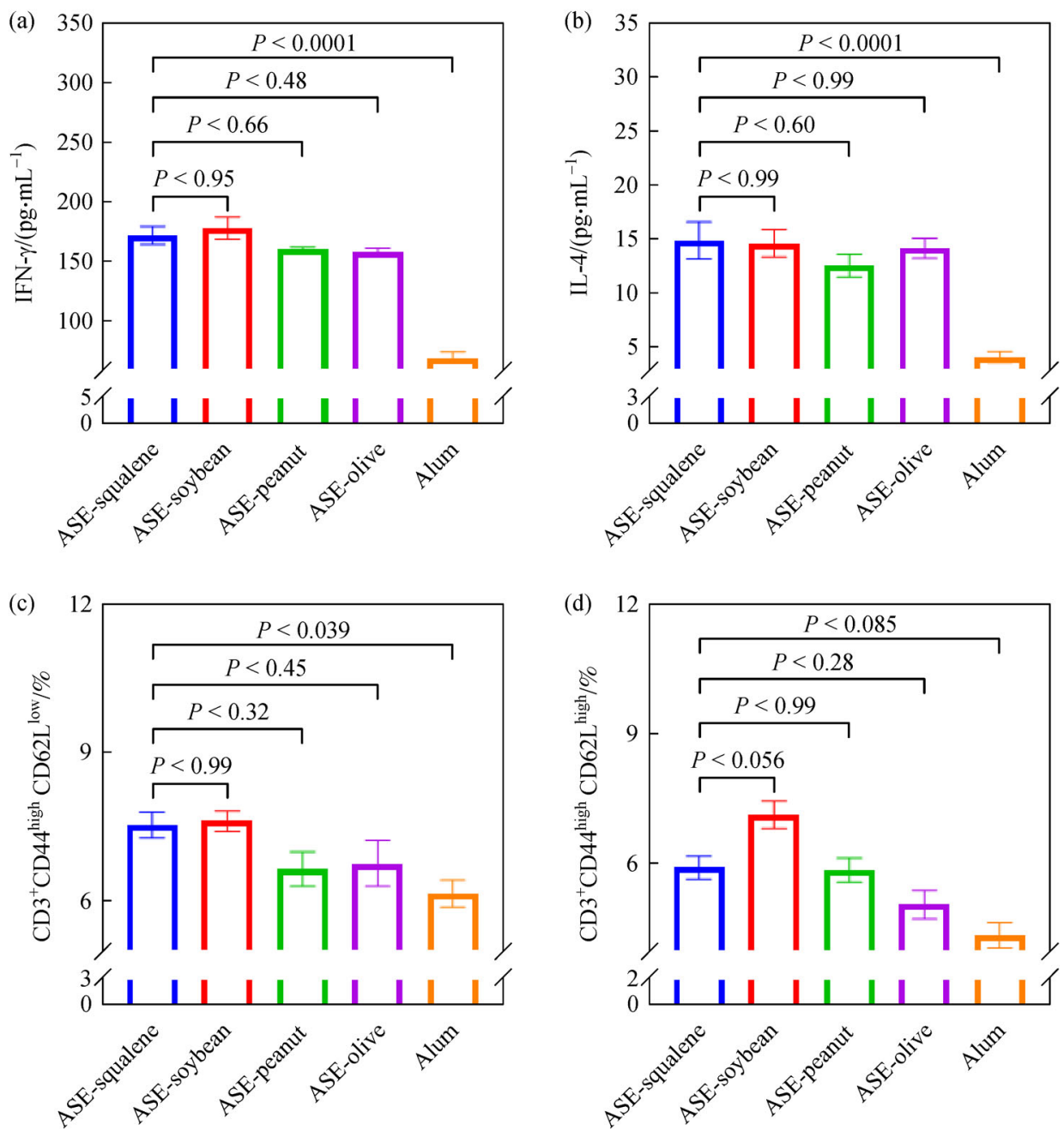

Fig. 4 Cytokine profile and memory T cell activations: (a) IFN- $\gamma$, (b) IL-4 levels in supernatant of ex vivo stimulated splenocytes, (c) effector memory T cells (CD44 ${ }^{\text {high }}$ CD62L $\left.{ }^{\text {low }}\right)$, and (d) central memory T cells $\left(\mathrm{CD} 44^{\text {high }}\right.$ CD62L $\left.\mathrm{L}^{\text {high }}\right)$ among CD3 ${ }^{+}$cells. Data were shown as mean \pm s.e.m. $(n=6)$ and analysed by one-way ANOVA.

suggesting a limited delayed-type hypersensitivity inflammation (Figs. 6(a) and 6(b)). Furthermore, the moderately low IL-10 concentration in all the ASE groups demonstrated the absence of immune tolerance during the onset of adaptive immunity (Fig. 6(c)). Consequently, all ASEs containing the various oils demonstrated a good biosafety profile and induced adequate immune activation as potential vaccine adjuvants.

\subsection{Effect of $\mathrm{O} / \mathrm{L}$ ratios on emulsion stability}

In the case of emulsion adjuvants, stability is always regarded as the elephant in the room. To study the storage stability of the ASEs, the emulsions were stored at $4{ }^{\circ} \mathrm{C}$ (storage temperature), $25{ }^{\circ} \mathrm{C}$ (room temperature), and $37^{\circ} \mathrm{C}$ (accelerating experiment), and monitored by optical microscopy and DLS (Fig. 7). The stability was determined by the particle size distribution, zeta potential distribution, and aggregation occurrence. All the ASEs were well dispersed at the beginning in Fig. 7(a), but ASEpeanut $(\mathrm{O} / \mathrm{L}=1.3: 1)$ and $\mathrm{ASE}-$ olive $(\mathrm{O} / \mathrm{L}=4: 1)$ tended to aggregate on Day 30, regardless of the temperature. Additionally, the ASE-soybean $(\mathrm{O} / \mathrm{L}=1: 2.75)$ remained stable for long-term storage. Additionally, the ASEsoybean was determined to have a relatively high absolute charge value $(-22.3667 \pm 2.5146 \mathrm{mV})$, indicating the increased electrostatic interaction between the droplets to promote stability by minimising droplet coalescence (Fig. S6, cf. ESM). Therefore, oils with a lower O/L ratio (soybean) may have a higher absolute zeta potential, contributing to their increased storage stability.

For antioxidant ability, we used an oxidation stability tester (892 Professional Rancimat, Metrohm) to determine the oxidation induction period of oil before and after emulsification. Oxygen was pumped into the oil and heated to $120{ }^{\circ} \mathrm{C}$ to accelerate the process. The oxidation induction period was monitored and calculated based on the conductivity changes of the samples. Oils with a higher 


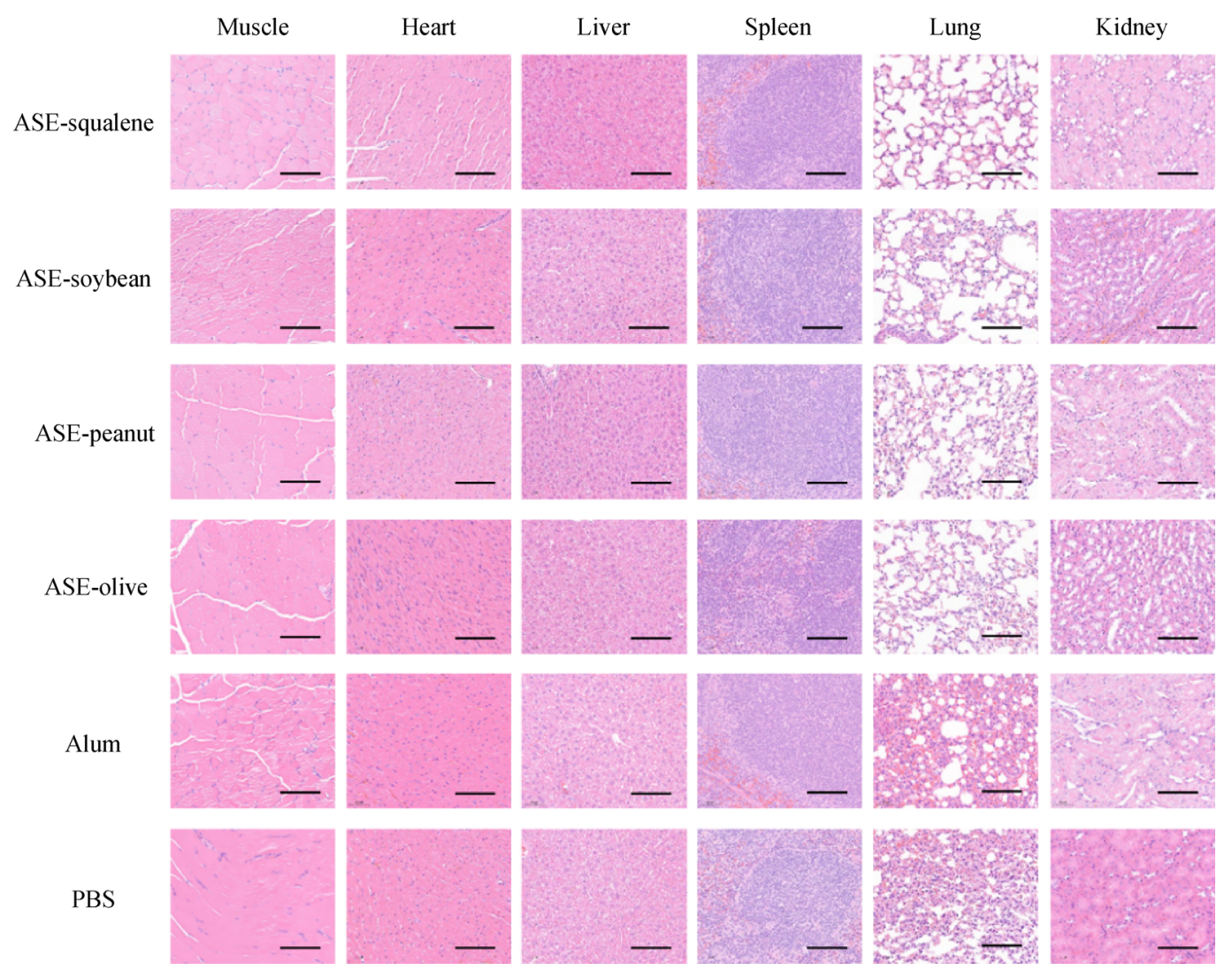

Fig. 5 Biocompatibility evaluations via H\&E staining of vital organ sections and injection sites (muscle) from BALB/c mice. Scale bar $=100 \mu \mathrm{m}$.

(a)

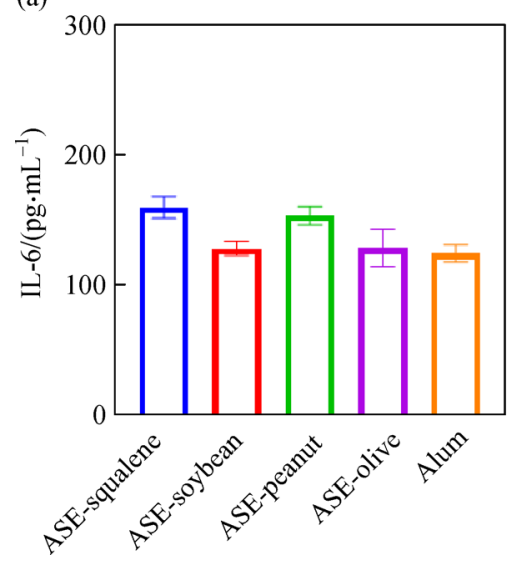

(b)

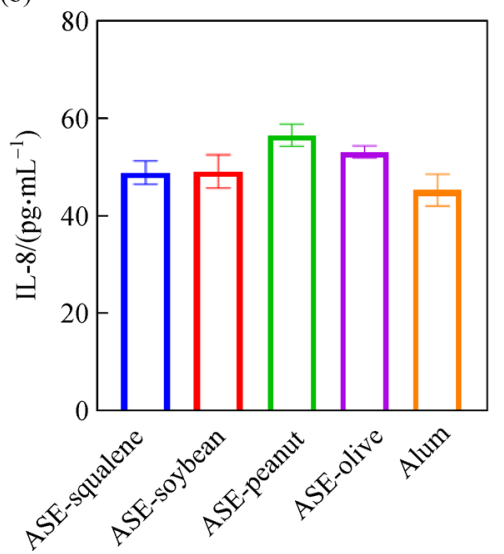

(c)

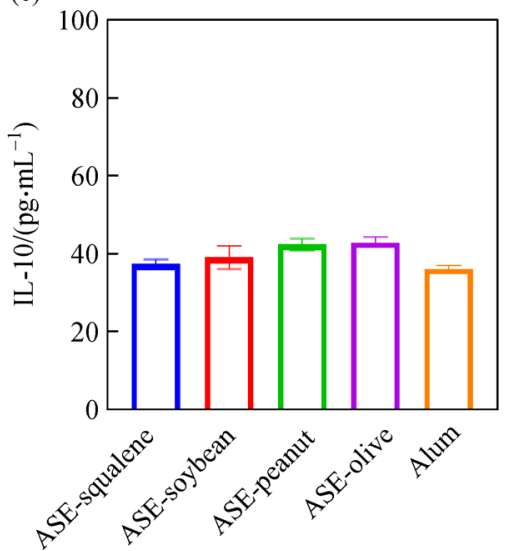

Fig. 6 (a) IL-6, (b) IL-8, and (c) IL-10 levels in supernatant of ex vivo stimulated splenocytes. Data were shown as mean \pm s.e.m. $(n=6)$ and analysed by one-way ANOVA.

$\mathrm{O} / \mathrm{L}$ ratio (olive oil) consumed a longer oxidation induction period, indicating enhanced oxidation stability, as illustrated in Table 5. Furthermore, ASEs were determined with relatively shorter oxidation induction periods, possibly due to the presence of alum ions in the solutions, which accelerated oil oxidation.

To comprehensively compare the stability of all the
ASEs, a Likert scale was developed [35,36]. Additionally, to quantify and characterise the stability, a brief stability evaluation scale among a group of nine random samples was designed based on the aggregation occurrence, size changes, and oxidation induction period (Table 2). ASEsoybean demonstrated a comparable SES store with ASEsqualene (Table 6 and Fig. 8), which was evidently higher 
Table 4 Biochemical parameters in the serum

\begin{tabular}{lccccc}
\hline Item & $\mathrm{ALT} /\left(\mathrm{U} \cdot \mathrm{L}^{-1}\right)$ & $\mathrm{ALP} /\left(\mathrm{U} \cdot \mathrm{L}^{-1}\right)$ & $\mathrm{LDH} /\left(\mathrm{U} \cdot \mathrm{L}^{-1}\right)$ & $\mathrm{BUN} /\left(\mathrm{U} \cdot \mathrm{L}^{-1}\right)$ & $\mathrm{AST} /\left(\mathrm{U} \cdot \mathrm{L}^{-1}\right)$ \\
\hline ASE-squalene & $60.5 \pm 25.0$ & $149.5 \pm 3.4$ & $711.0 \pm 135.7$ & $6.9 \pm 1.2$ & $79.5 \pm 18.2$ \\
ASE-soybean & $38.0 \pm 14.3$ & $189.0 \pm 17.4$ & $876.0 \pm 114.5$ & $7.9 \pm 1.7$ & $83.5 \pm 9.9$ \\
ASE-peanut & $76.5 \pm 31.5$ & $183.5 \pm 11.0$ & $878.0 \pm 56.1$ & $7.8 \pm 0.8$ & $89.5 \pm 16.1$ \\
ASE-olive & $70.0 \pm 53.4$ & $183.5 \pm 46.4$ & $929.0 \pm 379.2$ & $7.8 \pm 5.4$ & $91.5 \pm 36.5$ \\
Alum & $93.5 \pm 46.08$ & $178.5 \pm 18.2$ & $820.0 \pm 104.3$ & $4.9 \pm 1.0$ & $80.0 \pm 10.9$ \\
PBS & $64.0 \pm 17.5$ & $151.0 \pm 8.4$ & $730.0 \pm 71.4$ & $8.0 \pm 1.7$ & $94.5 \pm 23.5$ \\
\hline
\end{tabular}

\section{(a)}

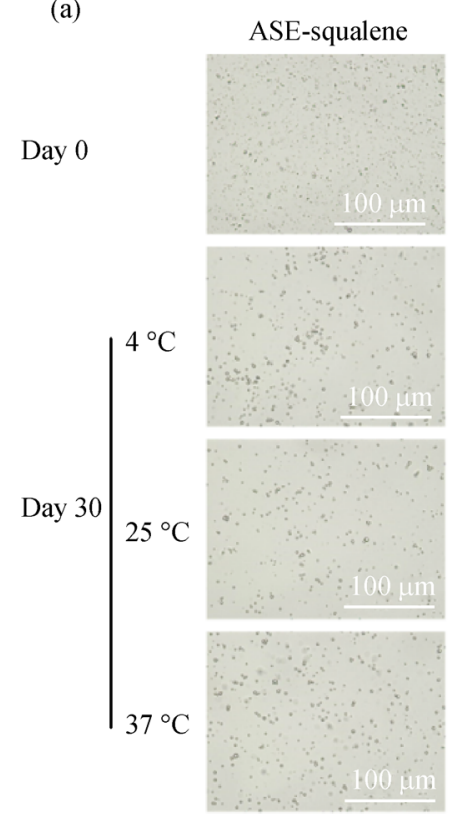

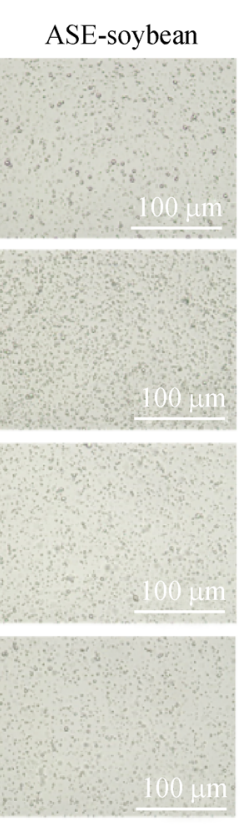

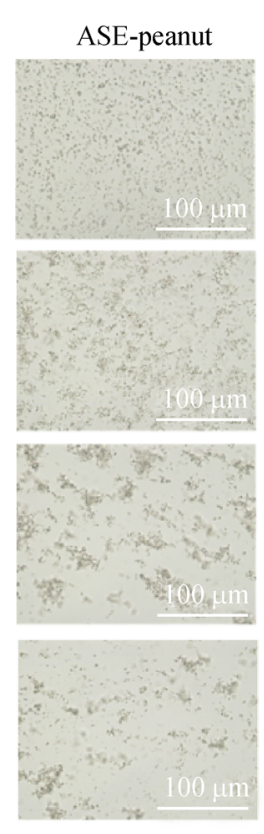

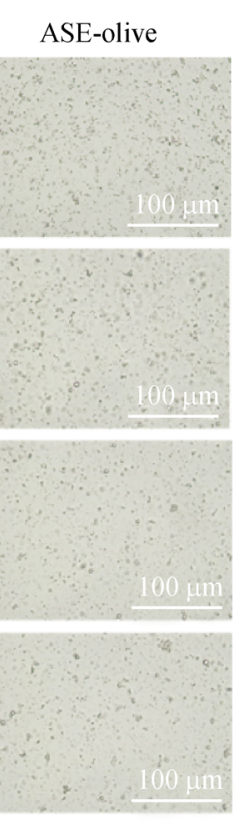
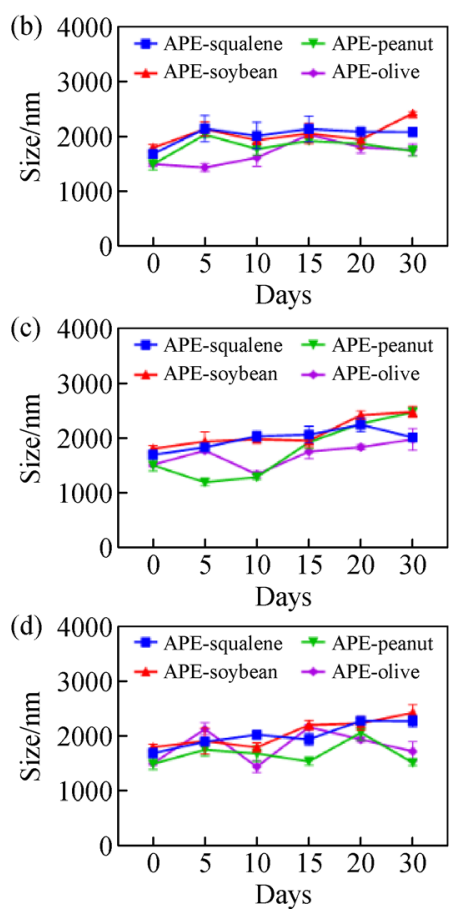

Fig. 7 Storage stability of ASEs. (a) Optical micrographs of ASEs at the indicated temperatures on 30 days. Scale bar $=100 \mu \mathrm{m}$. For optical microscopy determination, the images were acquired with $40 \times$ magnification. (b-d) Size of ASEs from Day 0 to Day 30 of storage at (b) $4{ }^{\circ} \mathrm{C}$, (c) $25^{\circ} \mathrm{C}$, and (d) $37^{\circ} \mathrm{C}$. Data were shown as the mean \pm s.e.m. $(n=3)$.

Table 5 Oxidation induction period of the oils and ASEs

\begin{tabular}{lcc}
\hline \multirow{2}{*}{ Oil/ASE } & \multicolumn{2}{c}{ Oxidation induction period/h } \\
\cline { 2 - 3 } & $0.830 \pm 0.005$ & $0.430 \pm 0.005$ \\
Squalene & $1.580 \pm 0.013$ & $0.630 \pm 0.010$ \\
Soybean & $1.690 \pm 0.013$ & $0.670 \pm 0.010$ \\
Peanut & $2.320 \pm 0.010$ & $0.900 \pm 0.150$ \\
Olive & & \\
\hline
\end{tabular}

than that of ASE-peanut and ASE-olive. Therefore, ASEsoybean, with a lower $\mathrm{O} / \mathrm{L}$ ratio, exhibited enhanced stability.

\section{Conclusions}

We optimised ASEs with similar sizes (approximately $1600.00 \pm 71.75 \mathrm{~nm}$ ) as a vaccine delivery system by controlling the alum concentration, continuous phase, and preparation parameters, tested the zeta potential, and captured the good dispersion of the ASEs under optical micrographs. The characteristics (such as size, zeta potential, and optical micrographs) of ASEs listed in Table 3, Fig. S6 and Fig. 7(a), which represent the ASEs we designed, were suitable for vaccine delivery. For stability evaluations, we used a Likert scale to comprehensively evaluate the stability of the emulsion adjuvants. Here, soybean oil, peanut oil, and olive oil showed increased antioxidant properties due to the presence of saturated fatty acids in the formulations. Furthermore, contrasting the low $\mathrm{O} / \mathrm{L}$ ratio oil phase (soybean oil), high $\mathrm{O} / \mathrm{L}$ ratios in the oil phases (olive oil or peanut oil) may increase the oxidation stability while decreasing the surface zeta potential to diminish long-term storage stability. Compared with squalene-based ASEs, soybeanbased ASEs induced comparable serum antibody levels, cytokine secretions, and safety profiles. ASE-olive and 
Table 6 Likert chart and factor analysis on the stability of the ASEs (mean \pm s.e.m.)

\begin{tabular}{lccccc}
\hline ASE & $\begin{array}{c}\text { Aggregation time of } \\
\text { micrograph }\end{array}$ & $\begin{array}{c}\text { Size change } \\
\text { degree }\end{array}$ & $\begin{array}{c}\text { Zeta change } \\
\text { degree }\end{array}$ & $\begin{array}{c}\text { Oxidation induction } \\
\text { period }\end{array}$ & $\begin{array}{c}\text { Total score } \\
\text { of stability }\end{array}$ \\
\hline ASE-squalene & $4.50 \pm 0.54$ & $4.17 \pm 0.75$ & $4.83 \pm 0.41$ & $1.670 \pm 0.005$ & $15.16 \pm 0.31$ \\
ASE-soybean & $4.17 \pm 0.75$ & $3.50 \pm 0.83$ & $4.17 \pm 0.41$ & $3.00 \pm 0.01$ & $14.83 \pm 0.37$ \\
ASE-peanut & $1.50 \pm 0.54$ & $1.83 \pm 0.98$ & $2.00 \pm 0.89$ & $3.00 \pm 0.01$ & $9.30 \pm 0.44$ \\
ASE-olive & $2.50 \pm 0.54$ & $2.17 \pm 0.98$ & $1.83 \pm 0.75$ & $4.00 \pm 0.15$ & $10.50 \pm 0.35$ \\
\hline
\end{tabular}

(a)

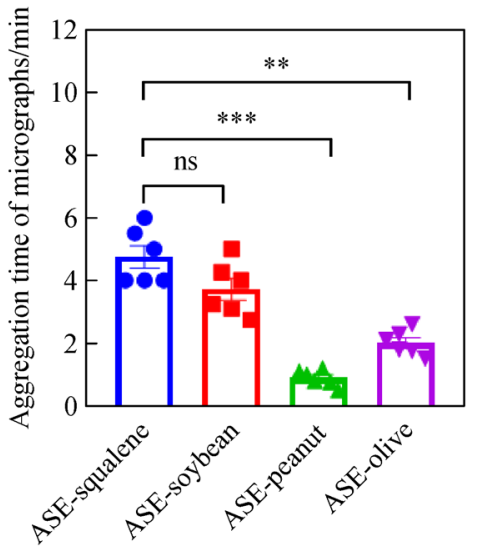

(b)

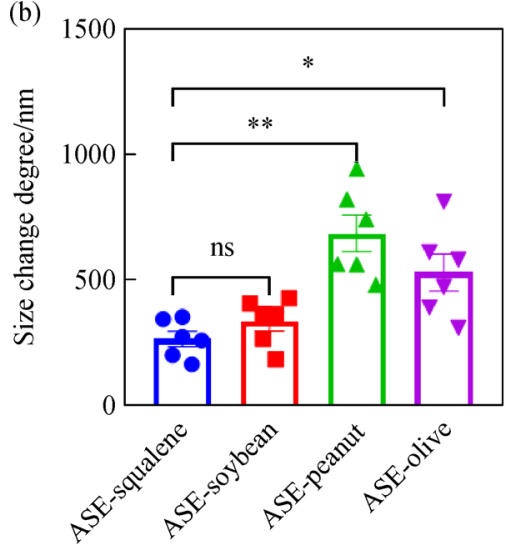

(c)

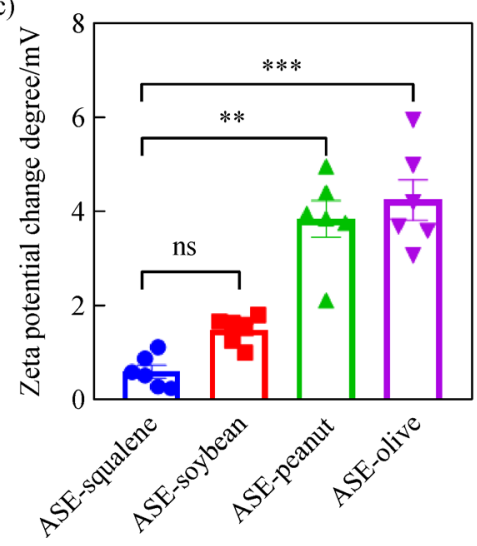

Fig. 8 Quantification and characterisation of the stability of ASEs: (a) aggregation time of micrographs, (b) size change degree, and (c) zeta potential change degree. Data were shown as the mean \pm s.e.m. $(n=9, * p<0.05, * * p<0.01, * * * p<0.001)$ and analysed by oneway ANOVA.

ASE-peanut treatments induced relatively lower immune responses. By presenting the parameters of the ASEs and comparing their stability and immunogenicity, soybean oil may represent the optimal oil phase for alum-stabilised emulsion adjuvants and may serve as a candidate oil phase for a stable, safe, and effective emulsion adjuvant.

Acknowledgements This work was supported by the Project supported by Beijing Nova Program of Beijing Municipal Science \& Technology Commission (Grant No. Z201100006820139), the CAS Project for Young Scientists in Basic Research (YSBR-010), the Pilot Project of Chinese Academy of Sciences (Grant No. XDB29040303), the Foundation for Innovative Research Groups of the National Natural Science Foundation of China (Grant No. 21821005), "From 0 to 1" Original Innovation Project of Basic Frontier Scientific Research Program of Chinese Academy of Sciences
(Grant No. 2020000071), Youth Project of National Natural Science Foundation of China (Grant No. 21908229), Youth Innovation Promotion Association of the Chinese Academy of Sciences (Grant No. 2020000053).

Compliance with Ethics Guidelines All animal protocols were approved by the Institutional Animal Care and Use. Committees at the Institute of Process Engineering, Chinese Academy of Sciences (approval ID: IPEAECA20210402). This study was performed in strict accordance with the Regulations for the Care and Use of Laboratory Animals and Guideline for Ethical Review of Animal (China, GB/T35892-2018). The authors modified all the techniques and procedures to provide for maximum comfort and minimal stress to the animals.

Electronic Supplementary Material Supplementary material is available in the online version of this article at https://dx.doi.org/10.1007/s11705-0212123-1 and is accessible for authorized users. 


\section{References}

1. Bosch F X, Robles C, Díaz M, Arbyn M, Baussano I, Clavel C, Ronco G, Dillner J, Lehtinen M, Petry K U, et al. HPV-Faster: broadening the scope for prevention of HPV-related cancer. Nature Reviews. Clinical Oncology, 2016, 13(2): 119-132

2. Zhao H, Zhou X Y, Zhou Y H. Hepatitis B vaccine development and implementation. Human Vaccines \& Immunotherapeutics, 2020, 16(7): 1533-1544

3. Zeng Z, Cheng L, Chen X. Progress in research on polio vaccine. Chinese Journal of Biologicals, 2019, 32(6): 713-716, 720 (in Chinese)

4. Bellini C, Horvati K. Recent advances in the development of protein-and peptide-based subunit vaccines against tuberculosis. Cells, 2020, 9(12): 2673

5. Cossette B, Kelly S H, Collier J H. Intranasal subunit vaccination strategies employing nanomaterials and biomaterials. ACS Biomaterials Science \& Engineering, 2021, 7(5): 1765-1779

6. Do Tien D, Kim H, Jeong J, Park K H, Yang S, Oh T, Kim S, Kang I, Chae C. Comparative evaluation of the efficacy of commercial and prototype PRRS subunit vaccines against an HP-PRRSV challenge. Journal of Veterinary Medical Science, 2018, 80(9): 1463-1467

7. Nevagi R J, Skwarczynski M, Toth I. Polymers for subunit vaccine delivery. European Polymer Journal, 2019, 114: 397-410

8. Chao L, Xu L, Song G, Zhuang L. Emerging nanomedicine approaches fighting tumor metastasis: animal models, metastasistargeted drug delivery, phototherapy, and immunotherapy. Chemical Society Reviews, 2016, 45(22): 6250-6269

9. Dupuis M, Denis-Mize K, Labarbara A, Peters W, Charo I, Mcdonald D, Ott G. Immunization with the adjuvant MF59 induces macrophage trafficking and apoptosis. European Journal of Immunology, 2015, 31(10): 2910-2918

10. Bui C, Bethmont A, Chughtai A, Gardner L, Sarkar S, Hassan S, Seale H, Macintyre C R. A systematic review of the comparative epidemiology of avian and human influenza A H5N1 and H7N9 - essons and unanswered questions. Transboundary and Emerging Diseases, 2016, 63(6): 602-620

11. Shah R R, Taccone M, Monaci E, Brito L A, Bonci A, O'Hagan D T, Amiji M M, Seubert A. The droplet size of emulsion adjuvants has significant impact on their potency, due to differences in immune cell-recruitment and-activation. Scientific Reports, 2019, 9(1): 11520

12. Singh Y, Meher J G, Raval K, Khan F A, Chaurasia M, Jain N K, Chourasia M K. Nanoemulsion: concepts, development and applications in drug delivery. Journal of Controlled Release, 2017, 252: $28-49$

13. Xia Y, Wu J, Du Y, Miao C, Ma G. Bridging systemic immunity with gastrointestinal immune responses via oil-in-polymer capsules. Advanced Materials, 2018, 30(31): 1801067

14. Peng S, Cao F, Xia Y, Gao X, Dai L, Yan J, Ma G. COVID-19 vaccines: particulate alum via Pickering emulsion for an enhanced COVID-19 vaccine adjuvant. Advanced Materials, 2020, 32(40): e2004210

15. Song T, Xia Y, Du Y, Chen M W, Qing H, Ma G. Engineering the deformability of albumin-stabilized emulsions for lymph-node vaccine delivery. Advanced Materials, 2021, 33(26): e2100106

16. Xia Y, Jie W, Wei W, Du Y, Tao W, Ma X, An W, Guo A, Miao C, Hua Y. Exploiting the pliability and lateral mobility of Pickering emulsion for enhanced vaccination. Nature Materials, 2018, 17(2): 187-194

17. Shimizu N, Ito J, Kato S, Eitsuka T, Nakagawa K. Significance of squalene in rice bran oil and perspectives on aqualene oxidation. Journal of Nutritional Science and Vitaminology, 2019, 65(Suppl.): S62-S66

18. Larsson $\mathrm{K}$, Istenic $\mathrm{K}$, Wulff $\mathrm{T}$, Jonsdottir $\mathrm{R}$, Kristinsson $\mathrm{H}$, Freysdottir J, Undeland I, Jamnik P. Effect of in vitro digested cod liver oil of different quality on oxidative, proteomic and inflammatory responses in the yeast Saccharomyces cerevisiae and human monocyte-derived dendritic cells. Journal of the Science of Food and Agriculture, 2015, 95(15): 3096-3106

19. Castelli G, Bianco I D, Kiyomi Mizutamari R. Polyphenol content in argentinean commercial extra virgin olive oil. European Journal of Lipid Science and Technology, 2018, 120(12): 1800124

20. Li Q, Tang X, Lu S, Wu J. Composition and tocopherol, fatty acid, and phytosterol contents in micro-endosperm ultra-high oil corn. Grasas y Aceites, 2019, 70(3): e311

21. Zhang T, Wang T, Liu R, Chang M, Jin Q, Wang X. Chemical characterization of fourteen kinds of novel edible oils: a comparative study using chemometrics. LWT, 2020, 118: 108725

22. Combs R, Bilyeu K. Novel alleles of FAD2-1A induce high levels of oleic acid in soybean oil. Molecular Breeding, 2019, 39(6): 79-90

23. Davis J P, Price K, Dean L L, Sweigart D S, Cottonaro J, Sanders T H. Peanut oil stability and physical properties across a range of industrially relevant oleic acid/linoleic acid ratios. Peanut Science, 2016, 43(1): PS14-17.1

24. Gnoni A, Longo S, Damiano F, Gnoni G V, Giudetti A M. Oleic acid and olive oil polyphenols downregulate fatty acid and cholesterol synthesis in brain and liver cells. In: Olives and Olive Oil in Health and Disease Prevention. London: Elsevier, 2021, 651657

25. Cooper J F, Weary C E, Jordan F T. The impact of non-endotoxin LAL-reactive materials on Limulus amebocyte lysate analyses. PDA Journal of Pharmaceutical Science and Technology, 1997, 51(1): $2-6$

26. Symoniuk E, Ratusz K, Krygier K. Oxidative stability and the chemical composition of market cold-pressed linseed oil. European Journal of Lipid Science and Technology, 2017, 119(11): 1700055

27. Siegler A J, Wiatrek S, Mouhanna F, Amico K R, Dominguez K, Jones J, Patel R R, Mena L A, Mayer K H. Validation of the HIV pre-exposure prophylaxis stigma scale: performance of Likert and semantic differential scale versions. AIDS and Behavior, 2020, 24(9): 2637-2649

28. Fan B, Fenton O, Daly K, Ding J, Chen Q, Chen Q. Alum split applications strengthened phosphorus fixation and phosphate sorption in high legacy phosphorus calcareous soil. Journal of Enviromental Sciences, 2021, 101: 87-97

29. Tan H, Han L, Yang C. Effect of oil type and $\beta$-carotene incorporation on the properties of gelatin nanoparticle-stabilized pickering emulsions. LWT, 2021, 141: 110903

30. Yao X X, Liu Z, Ma M Z, Chao Y C, Gao Y X, Kong T T. Control of particle adsorption for stability of Pickering emulsions in micro- 
fluidics. Small, 2018, 14(37): e1802902

31. Ghimire T R, Benson R A, Garside P, Brewer J M. Alum increases antigen uptake, reduces antigen degradation and sustains antigen presentation by DCs in vitro. Immunology Letters, 2012, 147(1-2): $55-62$

32. Carrillo J, Izquierdo-Useros N, Vila-Nieto C, Pradenas E, Blanco J. Humoral immune responses and neutralizing antibodies against SARS-CoV-2: implications in pathogenesis and protective immunity. Biochemical and Biophysical Research Communications, 2021, 538: 187-191

33. Lin J Z, Xu R, Tian X H. Threshold dynamics of an HIV-1 model with both viral and cellular infections, cell-mediated and humoral immune responses. Mathematical Biosciences and Engineering, 2019, 16(1): 292-319

34. Jalkanen S, Salmi M. Lymphatic endothelial cells of the lymph node. Nature Reviews. Immunology, 2020, 20(9): 566-578

35. Koksel Y, Gencturk M, Spano A, Reynolds M, Roshan S, Caycı Z. Utility of Likert scale (Deauville criteria) in assessment of chemoradiotherapy response of primary oropharyngeal squamous cell cancer site. Clinical Imaging, 2019, 55: 89-94

36. Krzych L J, Lach M, Joniec M, Cisowski M, Bochenek A. The Likert scale is a powerful tool for quality of life assessment among patients after minimally invasive coronary surgery. Kardiochir Torakochirurgia Pol, 2018, 15(2): 130-134 